\title{
Skill Evaluation in Women's Volleyball
}

Lindsay W. Florence

Gilbert W. Fellingham

gwf@byu.edu

Pat R. Vehrs

Nina P. Mortensen

Follow this and additional works at: https://scholarsarchive.byu.edu/facpub

Part of the Statistics and Probability Commons

\section{Original Publication Citation}

Florence, Lindsay W. Fellingham, Gilbert W. Vehrs, Pat R. and Mortensen, Nina P. (28) "Skill

Evaluation in Women's Volleyball," Journal of Quantitative Analysis in Sports: Vol. 4 : Iss. 2, Article 14.

\section{BYU ScholarsArchive Citation}

Florence, Lindsay W.; Fellingham, Gilbert W.; Vehrs, Pat R.; and Mortensen, Nina P., "Skill Evaluation in Women's Volleyball" (2008). Faculty Publications. 193.

https://scholarsarchive.byu.edu/facpub/193 


\title{
Journal of Quantitative Analysis in Sports
}

\section{Skill Evaluation in Women's Volleyball}

\author{
Lindsay W. Florence* \\ Gilbert W. Fellingham ${ }^{\dagger}$ \\ Pat R. Vehrs ${ }^{\ddagger}$ \\ Nina P. Mortensen**
}

*Brigham Young University, lwflorence@gmail.com

†Brigham Young University, gwf@byu.edu

${ }^{\ddagger}$ Brigham Young University, pat_vehrs@byu.edu

**Brigham Young University, nmvolleyset@ hotmail.com

Copyright (C) 2008 The Berkeley Electronic Press. All rights reserved. 


\title{
Skill Evaluation in Women's Volleyball
}

\author{
Lindsay W. Florence, Gilbert W. Fellingham, Pat R. Vehrs, and Nina P. \\ Mortensen
}

\begin{abstract}
The Brigham Young University Women's Volleyball Team recorded and rated all skills (pass, set, attack, etc.) and recorded rally outcomes (point for BYU, rally continues, point for opponent) for the entire 2006 home volleyball season. Only sequences of events occurring on BYU's side of the net were considered. Events followed one of these general patterns: serve-outcome, pass-setattack-outcome, or block-dig-set-attack-outcome. These sequences of events were assumed to be first-order Markov chains where the quality of each contact depended only on the quality of the previous contact but not explicitly on contacts further removed in the sequence. We represented these sequences in an extensive matrix of transition probabilities where the elements of the matrix were the probabilities of moving from one state to another. Each row of the count matrix, consisting of the number of times play moved from one transition state to another during the season, was assumed to have a multinomial distribution. A Dirichlet prior was formulated for each row, so posterior estimates of the transition probabilities were then available using Gibbs sampling. The different paths in the transition probability matrix were followed through the possible sequences of events at each step of the MCMC process to compute the posterior probability density that a perfect pass results in a point, a perfect set results in a point, etc. These posterior probability densities are used to address questions about skill performance in BYU Women's Volleyball.
\end{abstract}

KEYWORDS: volleyball, Markov chain, transition matrix, Markov chain Monte Carlo, Gibbs sampling, multinomial distribution, Bayesian Model 


\section{Introduction}

The Brigham Young University Women's Volleyball Team, a Division I intercollegiate team, used a notational analysis system to measure skill performance during the 2006 home volleyball season; every serve, pass, attack, and dig was recorded and graded in real time, while sets were graded after viewing the matches on film. Every touch made by the team was graded on a scale ranging as fine as $0-5$ points in order to quantify how well the skill was performed.

We assumed the sequences of hits followed a first-order Markov chain, where the quality of each hit depended only on the quality of the previous contact and not explicitly on contacts further removed in the sequence. We assumed a multinomial likelihood distribution for each row in the count matrix and a Dirichlet prior distribution for the associated probabilities. The count matrix consisted of the number of times play moved from one transition state to another during the season. The posterior distribution for the probabilities in each row is then proportional to the product of the likelihood and prior distributions. Gibbs sampling was implemented to calculate the posterior distributions of the probabilities of moving from one state to another. We used the mean of the posterior distribution of the probability as a point estimate to insert into the transition matrix. The transition probability matrix can then be used to estimate probabilities of various sequences of events. We used the transition probability matrix to estimate the unconditional probabilities associated with performing a particular skill at various levels.

Section 2 examines previous work on volleyball analysis and estimating transition probabilities. Section 3 discusses the data and the notational grading system used for the BYU Women's Volleyball Team. Section 4 discusses the transitional probability matrix, our Bayesian model, and the methods used to calculate posterior distributions of unconditional probabilities for a certain skill resulting in a point for BYU, continuation of rally, or a point for the opponent. Section 5 presents the resulting point estimates and posterior distributions for the unconditional probabilities. Section 6 discusses ways the methodology might be used to improve play.

\section{Previous Literature}

In order to fully comprehend a sport team's performance, the skills used must be recorded, graded and analyzed quantitatively. According to Daniel and Hughes (2003), there has not been a considerable amount of quantitative analyses published concerning the performance of volleyball skills. However, various 
notational analysis systems have been developed for the purpose of analyzing volleyball skills (Coleman et al. 1971; Coleman 1975; Sawula 1977; Lirdla 1980; Vojik 1980; Rose 1983; Eom and Schutz 1992; Zetou et al. 2007). This paper builds on this previous work and adds an extra dimension: although notational systems have long been used to quantify volleyball performance in some dimensions, there has never been an extensive attempt to include the grading of setting in the systems. By grading setting independent of the attack and outcome, the natural association between the performance of one skill and the performance of subsequent skills can be examined. The data set we used was produced by and for the BYU Women's Volleyball Team and included a rating of every skill performed by team members during the 2006 home season.

Because volleyball skills are performed in a fairly rigid time sequence pattern (pass-set-attack, etc.), it seemed natural to treat these patterns as Markov chains. That is, we approached the problem as one of estimating the probability of transitioning from one state to another while the ball was on BYU's side of the net. Common methods used in estimating transition probabilities have included maximum likelihood (Anderson and Goodman 1957; Duncan and Lin 1972; Craig and Sendi 2002), Bayesian methods (Lee et al. 1968; Boender and Rinnooy-Kan 1983; Fahrmeir 1992; Assoudou and Essebbar 2003), least squares (Miller 1952; Telser 1963), weighted least squares (Madansky 1959), restricted least squares (Theil and Rey 1966; Lee et al. 1968), and weighted restricted least squares (Theil and Rey 1966). Lee et al. (1968) compared different methods of estimating transition probabilities including least squares, weighted least squares, maximum likelihood, and Bayesian models. They found that Bayesian estimators performed better than maximum likelihood, least squares, and weighted least squares estimators. These results were based on the mean square error and absolute value of the error from various nonparametric tests. Assoudou and Essebbar (2003) also found that Bayesian estimators performed better than maximum likelihood and had a lower mean square error for two- and three-state models.

Most work using Bayesian models to estimate transition probabilities has assumed a multinomial likelihood distribution and a Dirichlet prior distribution (Lee et al. 1968; Satia and Lave 1973; Ezzati 1974; Meshkani and Billard 1992; McKeigue et al. 2000; Ozekici and Soyer 2003; Zhao et al. 2005). The models used by Cargnoni et al. (1997) and Assoudou and Essebbar (2003) assumed different prior distributions including the normal distribution and Jeffreys' prior distribution, respectively. To calculate Bayesian point estimates of the transition probabilities, several earlier methods used the posterior mean or mode (Lee et al. 1968; Boender and Rinnooy-Kan 1983; Fahrmeir 1992; McKeigue et al. 2000). DeGroot (1970) showed that the posterior expectation 
Florence et al.: Skill Evaluation in Women's Volleyball

is the optimal Bayesian estimator with respect to the quadratic loss function.

\section{The Data}

The data were recorded into a program called Data Volley (Data Project, Salerno, Italy, release 2.1.9). The grading system was developed based on the number of possible codes Data Volley was capable of handling. Serves were graded on a six-point (0-5) scale, passes on a five-point (0-4) scale, and attacks by position of the court (middle, right side, left side, back row) and outcome (kill, rally continuation, error, block). We evaluated sets according to three variables: distance from the net ( $0-3$ feet, $3-5$ feet, etc.), height of the set (high and low), and position of the set in relation to the hitter (inside and outside). Digs and blocks were also noted in the data.

A trained member of the women's volleyball coaching staff graded and recorded in real time every serve, pass, dig, and attack performed by BYU for the 13 home matches during the 2006 season. A default code was inserted for sets, so these could be graded at a later time while viewing the game on film. To grade the sets, the matches were filmed by two cameras observing different angles of the court at the same time. One camera recorded the entire court from behind the end line of the BYU women's team. The other camera was parallel to and approximately five feet away from the net, showing only BYU's side of the court. Questionable sequences found in the data were also verified by viewing the sequences on film. The hits recorded for the opposing team included serves and attacks. This allowed us to track when the ball had crossed the net. The final data set consisted of over 7,300 touches of the ball for the BYU team.

Considerable work was necessary before the data were ready to analyze. The data set contained many unnecessary codes that had to be removed. The information in the data that was necessary for the analysis included the number of the player who made contact with the ball, the skill type and skill grade of the contact, and when the game ended. The team that contacted the ball could be determined by looking at the player's number, which was coded so BYU numbers were less than 50 and opposing team numbers were greater than 50. Although the score was inserted by the person coding the data, it was often inaccurate. To alleviate this problem, the score was determined at the conclusion of each rally by identifying the next team to serve. The outcome for the final rally of each game was determined by the final score.

Since only touches on BYU's side of the net were considered, continuation of the rally was determined by observing if the ball returned to BYU's side 
of the net during a rally. However, because the person recording the data was less interested in the opponent than BYU, sometimes there were no hits recorded for the opposing team in a specific sequence, making it appear as if the BYU team hit the ball more than three times in a row. Such sequences had to be located and corrected before the analysis could be performed.

\section{Methods}

Every time the ball was on BYU's side of the net, a sequence of events occurred that followed one of these patterns: serve-outcome, pass-set-attack-outcome, or dig-set-attack-outcome. The outcome was a point for BYU, a point for the opponent, or continuation of the rally. We assumed these sequences were firstorder Markov chains. We represented these sequences in a matrix of transition probabilities where the elements in the matrix represented the probabilities of moving from one state to another (e.g., a four-point pass to a perfect set). Impossible sequences (e.g., a perfect pass to an ace serve) were constrained to have zero probability. Sequences that always occurred (e.g., an attack kill to a point for BYU) were assigned a probability of one. Because setting had two measurements recorded, we calculated the transition probability matrix including set distance from net, set placement, or both according to the measurement we wanted to analyze.

The transition matrix was comprised of 35 states when analyzing set distance, 37 states with set placement, and 55 states with combined set distance and placement. The states specified in the matrix were: one opponent serve; six BYU float serves; six BYU jump serves; six passing types; five set distances, seven set placements, or 25 combinations of set distance and placement; seven attack types; one dig type; and three outcomes.

We used a Bayesian paradigm to model the unknown transition probabilities. We assumed a multinomial likelihood

$$
f\left(y_{i 1}, \ldots, y_{i k} \mid \pi_{i 1}, \ldots, \pi_{i k}\right) \propto \pi_{i 1}^{y_{i 1}} \pi_{i 2}^{y_{i 2}} \ldots \pi_{i k}^{y_{i k}}
$$

for each row, $i=1, \ldots, m$, in the count matrix, where $k$ is the number of possible states that could occur next in the sequence of touches and $m$ is the number of states in the transition matrix. The probability of moving from state $i$ to another state $j$ in the transition probability matrix is represented by $\pi_{i j}$, where $\sum_{j=1}^{k} \pi_{i j}=1$. The data $y_{i j}$ consist of the number of times play moved from state $i$ to another state $j$ during the season. The count matrix is comprised of all the $y_{i j}$ 's. 
We specified our prior probability densities in each row to be distributed as Dirichlet random variables

$$
f\left(\pi_{i 1}, \ldots, \pi_{i k} \mid \alpha_{i 1}, \ldots, \alpha_{i k}\right) \propto \pi_{i 1}^{\alpha_{i 1}-1} \pi_{i 2}^{\alpha_{i 2}-1} \ldots \pi_{i k}^{\alpha_{i k}-1}
$$

where each $\alpha_{i j}$ represents how often we expected the women's team to move from state $i$ to state $j$ relative to moving to a different state in the transition probability matrix. Prior counts were determined by one of the authors, a former volleyball coach. To check for sensitivity to prior assumptions, we also ran an analysis with prior counts $\left(\alpha_{i j}\right)$ all equal to one. The results of the sensitivity analysis are discussed in Section 5.

We used Markov chain Monte Carlo methods to produce a posterior distribution

$$
f\left(\pi_{i 1}, \ldots, \pi_{i k} \mid y_{i 1}, \ldots, y_{i k}, \alpha_{i 1}, \ldots, \alpha_{i k}\right) \propto \pi_{i 1}^{y_{i 1}+\alpha_{i 1}-1} \pi_{i 1}^{y_{i 2}+\alpha_{i 2}-1} \ldots \pi_{i k}^{y_{i k}+\alpha_{i k}-1}
$$

for each row $i$ in the transition matrix. We used the mean of the posterior distribution, $\frac{y_{i j}+\alpha_{i j}}{\sum_{j=1}^{k}\left(y_{i j}+\alpha_{i j}\right)}$, for each of the $\pi_{i j}$ 's as point estimates to insert in the transition probability matrix.

In addition to estimating the transition probability matrix, we calculated the unconditional probabilities of moving from a certain state (e.g., a perfect pass) to an outcome (e.g., a point for BYU). To obtain a point estimate for the unconditional probability, we considered all possible sequences of touches that could occur between the state and outcome in the transition probability matrix. For each sequence, we multiplied the corresponding probabilities in the transition matrix. Using the law of total probability, we summed the probability of each sequence to get the unconditional probability of going from a certain state to an outcome.

In order to understand how much variability existed in our unconditional probability point estimates, we calculated the distribution for each unconditional probability using Gibbs sampling. To efficiently draw values from the posterior distribution, we drew $x_{1}, x_{2}, \ldots, x_{k}$ from independent gamma distributions with shape parameters $y_{i 1}+\alpha_{i 1}, y_{i 2}+\alpha_{i 2}, \ldots, y_{i k}+\alpha_{i k}$ and common scale parameter and calculated $\pi_{i j}=x_{j} / \sum_{j=1}^{k} x_{j}$ (Gelman et al. 2004). We computed a draw of the unconditional probability using the current state of the transition probability matrix at each step of the MCMC process. The unconditional probability distributions were based on 100,000 realizations from each row's posterior distribution. 


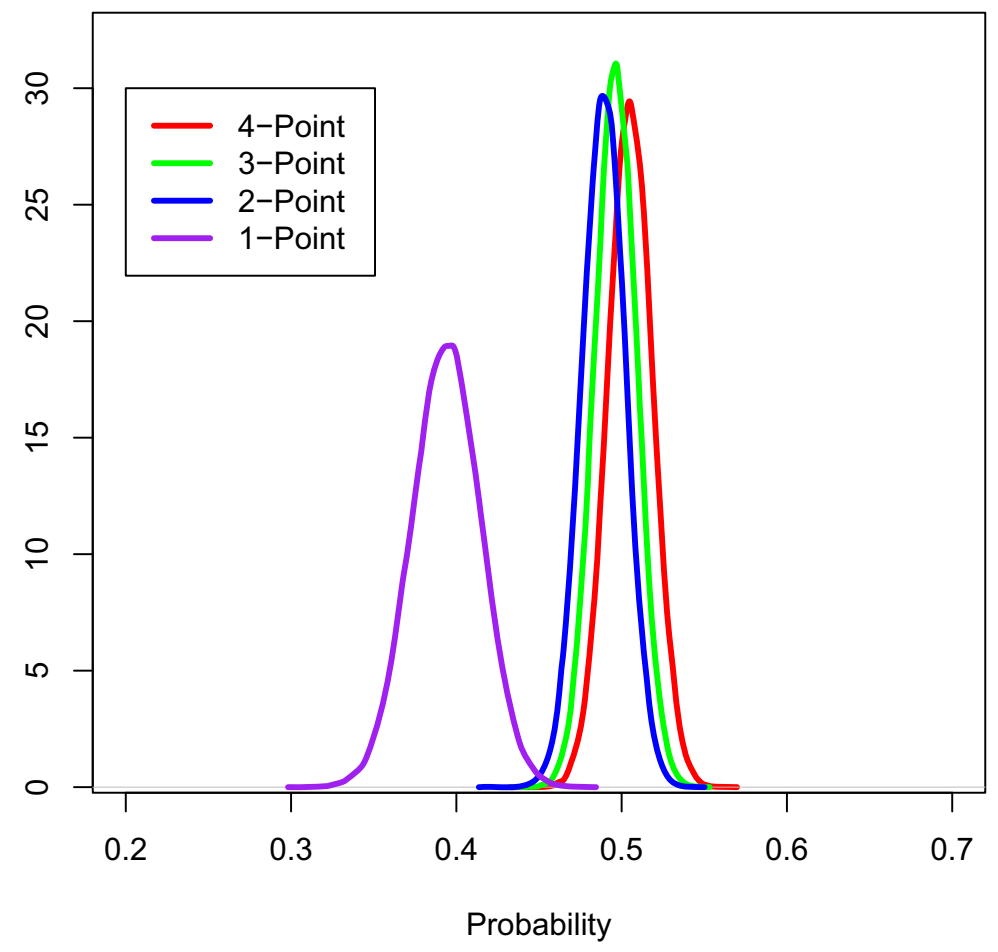

Figure 1: Posterior distributions for the unconditional probabilities of pass types leading to scoring a point.

\section{Results}

We summarize results by focusing on the unconditional probabilities of moving from a certain skill to a rally outcome. Figure 1 shows the posterior distributions for the unconditional probability of the present rally sequence ending in a point for BYU following a pass of the given point rating. A 0-point pass is not shown because it can never end in a point for BYU. Similarly, Figures 2 and 3 show the posterior distributions for the unconditional probabilities of various set types leading to an immediate point for BYU. Finally, Figure 4 shows the posterior distributions of the probability of attacks by position on the court leading to a point for BYU. Point estimates for these probabilities, as well as the probability of the rally continuing and a point being scored by the opposition, are shown in Table 1. 
Florence et al.: Skill Evaluation in Women's Volleyball

Table 1: The unconditional probability point estimates for pass types, sets certain distances from the net, set placements, and attack positions resulting in the various outcomes.

Pass Types

\begin{tabular}{|c|c|c|c|}
\hline Pass & Score Point & Continue Rally & Opponent Score \\
\hline 4-Point & 0.505 & 0.260 & 0.235 \\
3-Point & 0.496 & 0.259 & 0.245 \\
2-Point & 0.489 & 0.262 & 0.249 \\
1-Point & 0.394 & 0.278 & 0.328 \\
\hline
\end{tabular}

Sets Certain Distances from Net

\begin{tabular}{|c|c|c|c|}
\hline Set Distance & Score Point & Continue Rally & Opponent Score \\
\hline 0-3 Feet & 0.506 & 0.239 & 0.255 \\
3-5 Feet & 0.511 & 0.258 & 0.231 \\
5-8 Feet & 0.498 & 0.267 & 0.235 \\
8-10+ Feet & 0.426 & 0.293 & 0.281 \\
Set not by Setter & 0.456 & 0.290 & 0.254 \\
\hline
\end{tabular}

Set Placements

\begin{tabular}{|c|c|c|c|}
\hline Set Placement & Score Point & Continue Rally & Opponent Score \\
\hline Perfect & 0.509 & 0.259 & 0.232 \\
Low and Inside & 0.510 & 0.258 & 0.232 \\
High and Outside & 0.492 & 0.271 & 0.237 \\
Low and Outside & 0.495 & 0.260 & 0.245 \\
High and Inside & 0.472 & 0.284 & 0.244 \\
\hline
\end{tabular}

Attack Positions

\begin{tabular}{|c|c|c|c|}
\hline Attack & Score Point & Continue Rally & Opponent Score \\
\hline Middle & 0.530 & 0.243 & 0.227 \\
Right Side & 0.545 & 0.207 & 0.248 \\
Left Side & 0.495 & 0.283 & 0.222 \\
Back Row & 0.384 & 0.296 & 0.320 \\
\hline
\end{tabular}




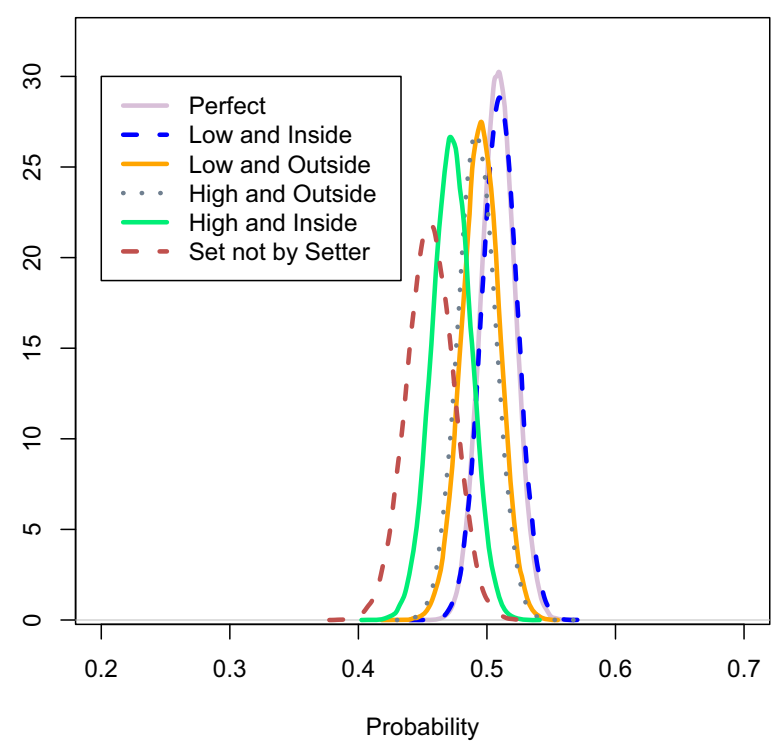

Figure 2: Posterior distributions for the unconditional probabilities of set placements leading to scoring a point.

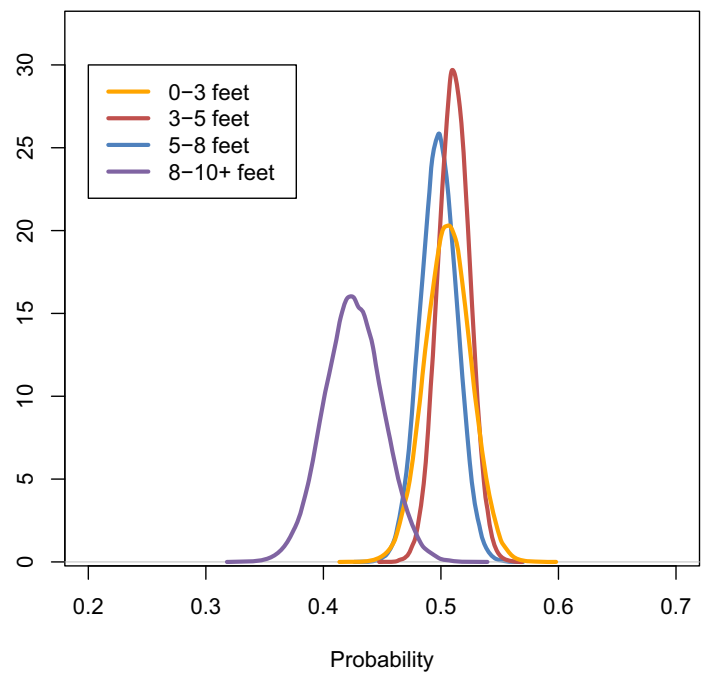

Figure 3: Posterior distributions for the unconditional probabilities of sets from various distances leading to scoring a point. 
Florence et al.: Skill Evaluation in Women's Volleyball

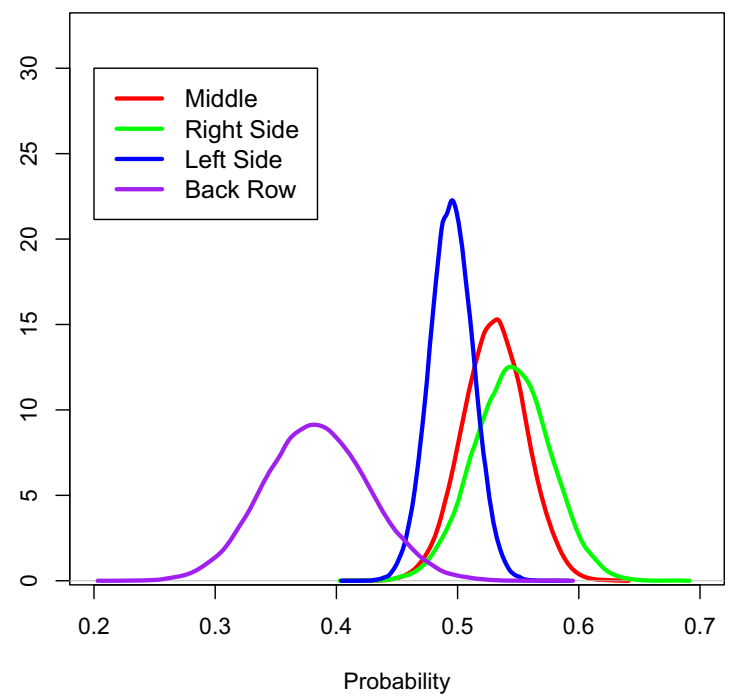

Figure 4: Posterior distributions of unconditional probabilities of attacks from various positions leading to scoring a point.

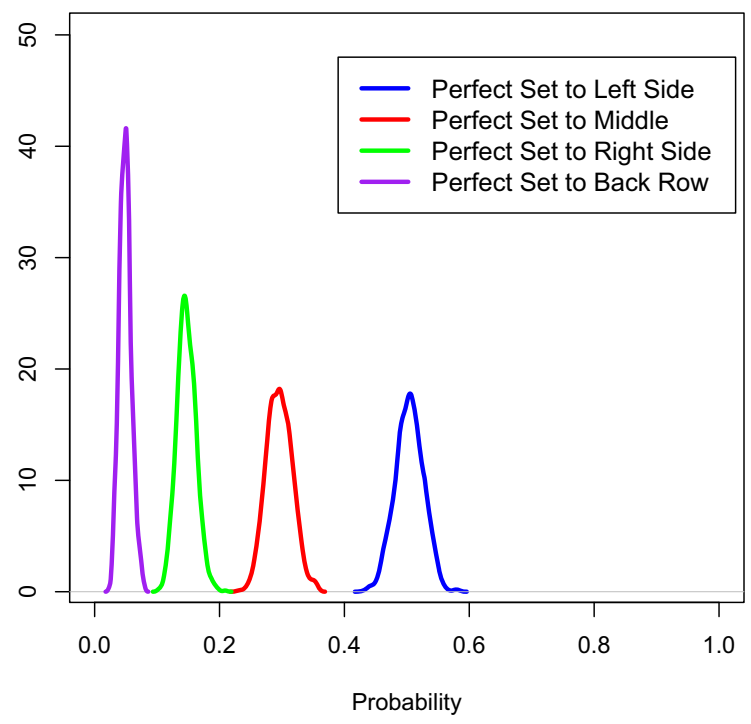

Figure 5: Posterior distributions of the transition probabilities of a perfect set to the various attack positions. 
We also performed a sensitivity analysis on the influence of the prior specification by setting the prior counts to 1 for every state where the transition probability was not constrained to be zero or one. The probabilities of passes with the various ratings leading to outcomes using these two prior distributions are shown in Figures 1 and 6 (posterior densities) and Tables 1 and 2 (point estimates). The outcomes are virtually indistinguishable for the two prior specifications. Thus, we have little reason to believe that the prior specifications we used had a marked influence on the posterior distributions. Similar differences were observed for all other unconditional probabilities.

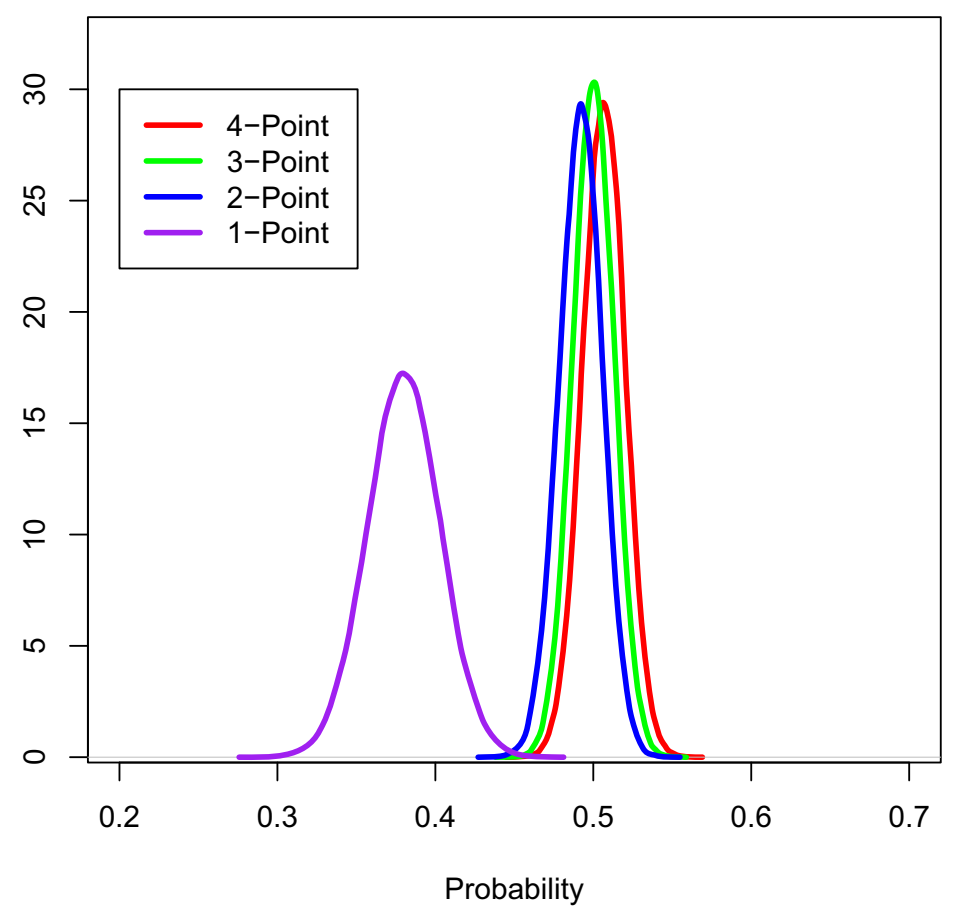

Figure 6: Posterior distributions for the unconditional probabilities of pass types leading to scoring a point when setting the prior counts to 1 for every state where the transition probability was not constrained to be 0 or 1 . 
Florence et al.: Skill Evaluation in Women's Volleyball

Table 2: Probability point estimates for passing to certain outcomes when prior counts were all assumed to be 1 .

\begin{tabular}{|c|c|c|c|}
\hline Pass Score & BYU Score & Continue Rally & Opponent Score \\
\hline 4-Point & 0.507 & 0.258 & 0.235 \\
3-Point & 0.500 & 0.257 & 0.243 \\
2-Point & 0.492 & 0.261 & 0.247 \\
1-Point & 0.380 & 0.279 & 0.341 \\
\hline
\end{tabular}

\section{Discussion}

We recognize that this analysis is applicable only to BYU women's volleyball. Nonetheless, it is not unreasonable to look for generalizations that might be applicable to other teams. We also recognize that there are many types of questions that could be asked based on the analysis that we have presented. We consider just four areas that may provide useful information for coaches.

Many coaches rate passers based on their passing average. This system seems to be problematic based on our results. The passing average assumes that the difference between a 1-point pass and a 2-point pass is equivalent to that between a 2-point pass and a 3-point pass, etc. This is obviously not the case. For example, a player with a 3.0 passing average who earns that average with equal numbers of 2-point, 3-point, and 4-point passes would have a point probability that the rally would terminate with a point for BYU of $\frac{1}{3}(.505+.496+.489)=.497$. Another player who earns a 3.0 passing average by having $70 \%$-point passes, $20 \% 1$-point passes, and 10\% 0-point passes would only have a point probability of $.7 * .505+.2 * .394=.432$. While these examples are admittedly extreme, the deficiencies of the average as a rating system for passers is obvious. The large discrepancy of probability of point production from 0-point and 1-point passes relative to 2-, 3-, and 4-point passes should be taken into account.

In a similar vein, it seems reasonable that the target a passer aims for should be moved further off the net; the penalty paid for a 2-point pass is small compared to that paid for an overpass. Sending a setter close to the net leads to the occasional spectacular play but, based on our analysis, would have a lower expected long-run return.

We now take a brief look at the back set or set to the right side of the court. Figure 5 shows the probability of a perfect set being made to the various attack points on the court. It is easy to see that the probability of making a perfect 
back set is much lower than the probability of making a perfect set either to the left side or to the middle of the court. However, based on the results shown in Figure 4, a strategy that avoids the back set because of its difficulty would not be wise. The right side attack has an excellent probability of ending a rally positively. The difficulty of making the set should be tempered by the results found in Figures 2 and 3. The penalty paid for a less than perfect set is not shown to be high in this analysis. If the high and inside delivery can be avoided (Figure 2) the attack has a good probability of being successful.

Finally, we note that, for the BYU women's team, at least, the back row set should be avoided. This attack has significantly lower probability of success (Figures 3 and 4). We conjecture that this result would generalize well to other women's teams, but have some doubt about applying this generalization to men's teams.

We believe that the methodology described in this paper can be used to assist a coach in allocating practice time, focusing on optimal skill development, and optimizing attack strategies. It seems likely that extensions of this method could be implemented to help a coach determine which players (and the skill sets they bring to the court) should be used to form an optimal team.

\section{References}

Anderson, T. W. and Goodman, L. A. (1957), "Statistical Inference about Markov Chains," The Annals of Mathematical Statistics, 28, 89-110.

Assoudou, S. and Essebbar, B. (2003), "A Bayesian Model for Markov Chains via Jeffrey's Prior," Communications in Statistics, 32, 2163-2184.

Boender, C. G. E. and Rinnooy-Kan, A. H. G. (1983), "A Bayesian Analysis of the Number of Cells of a Multinomial Distribution," The Statistician, 32, 240-248, proceedings of the 1982 I.O.S. Annual Conference on Practical Bayesian Statistics.

Cargnoni, C., Muller, P., and West, M. (1997), "Bayesian Forecasting of Multinomial Time Series Through Conditionally Gaussian Dynamic Models," Journal of the American Statistical Association, 92, 640-647.

Coleman, J. (1975), "A statistical evaluation of selected volleyball techniques at the 1974 world's volleyball championships," Ph.D. thesis, Brigham Young University, Provo, Utah. 
Coleman, J., Neville, B., and Gordon, B. (1971), "A statistical system for volleyball and its use in Chicago women's association," International Volleyball Review, 27, 72-73.

Craig, B. A. and Sendi, P. P. (2002), "Estimation of the transition matrix of a discrete-time Markov chain," Health Economics, 11, 33-42.

Daniel, R. and Hughes, M. (2003), "Playing patterns of elite and non-elite volleyball," Journal of Sports Sciences (London), 21, 268.

DeGroot, M. (1970), Optimal Statistical Decisions, New York: McGraw-Hill Inc.

Duncan, G. T. and Lin, L. G. (1972), "Inference for Markov Chains Having Stochastic Entry and Exit," Journal of the American Statistical Association, 67, 761-767.

Eom, H. and Schutz, R. (1992), "Statistical analyses of volleyball team performance," Research Quarterly for Exercise and Sport, 63, 11-18.

Ezzati, A. (1974), "Forecasting Market Shares of Alternative Home-Heating Units by Markov Process using Transition Probabilities Estimated from Aggregate Time Series Data," Management Science, 21, 462-473.

Fahrmeir, L. (1992), "Posterior Mode Estimation by Extended Kalman Filtering for Multivariate Dynamic Generalized Linear Models," Journal of the American Statistical Association, 87, 501-509.

Gelman, A., Carlin, J., Stern, H., and Rubin, D. (2004), Bayesian Data Analysis, Chapman \& Hall, 2nd ed.

Lee, T. C., Judge, G. G., and Zellner, A. (1968), "Maximum Likelihood and Bayesian Estimation of Transition Probabilities," Journal of the American Statistical Association, 63, 1162-1179.

Lirdla, D. (1980), "Statistical study of individual ball play," Volleyball Technical Journal, 5, 31-34.

Madansky, L. (1959), "Least Squares Estimation in Finite Markov Processes," Psychometrika, 24, 137-144.

McKeigue, P. M., Carpenter, J. R., Parra, E. J., and Shriver, M. D. (2000), "Estimation of admixture and detection of linkage in admixed populations by a Bayesian approach: application to African-American populations," Annals of Human Genetics, 64, 171-186. 
Meshkani, M. R. and Billard, L. (1992), "Empirical Bayes Estimators for a Finite Markov Chain," Biometrika, 79, 185-193.

Miller, G. (1952), "Finite Markov Processes in Psychology," Psychometrika, $17,149-167$.

Ozekici, S. and Soyer, R. (2003), "Network Reliability Assessment in a Random Environment," Naval Research Logistics, 50, 574-591.

Rose, R. (1983), "Statistical analysis at the 1983 Men's N.C.A.A. National Championship," Volleyball Technical Journal, 7, 15-17.

Satia, J. K. and Lave, R. E. (1973), "Markovian Decision Processes with Uncertain Transition Probabilities," Operations Research, 21, 728-740.

Sawula, L. (1977), "Individual action plan," Volleyball Technical Journal, 3, $2-8$.

Telser, L. (1963), Measurement of Economics, Stanford: Stanford University Press.

Theil, H. and Rey, G. (1966), "A Quadratic Programming Approach to the Estimation of Transition Probabilities," Management Science, 12, 714-721.

Vojik, J. (1980), "Several remarks to a system of accumulating data in volleyball," Volleyball Technical Journal, 5, 35-41.

Zetou, E., Moustakidis, A., Tsigilis, N., and Komninakidou, A. (2007), "Does Effectiveness of Skill in Complex I Predict Win in Men's Olympic Volleyball Games?" Journal of Quantitative Analysis in Sports, 3, article 3.

Zhao, J. X., Foulkes, A. S., and George, E. I. (2005), "Exploratory Bayesian Model Selection for Serial Genetics Data," Biometrics, 61, 591-599. 